\title{
The emerging clinical relevance of genomic profiling in neuroendocrine tumours
}

\author{
Guney Isa Burak ${ }^{1 \dagger}$, Sonmezler Ozge ${ }^{2 \dagger}$, Mujde Cem², Buyukdereli Gulgun', Dogruca Yapar Zeynep ${ }^{1}$ and Bisgin Atil ${ }^{3^{*}}$
}

\begin{abstract}
Background: Neuroendocrine tumours (NETs) arise from hormone-producing or nervous system cells and can develop from anywhere in the body. They have heterogeneous origins from skin to gastrointestinal track and a complicated histology. Thus, there is an inevitable need for genomic profiling to determine the exact genetics of each tumour for prognosis and treatment strategies to overcome the disease's complexity. For this purpose, nextgeneration-sequencing (NGS) is the most reliable methodology for both germ-line and somatic studies to make a clinical diagnosis. In this study, we analyse liquid biopsies, formalin fixed paraffin embedded (FFPE) tissues, and peripheral blood samples for their ability to provide information for actionability.

Methods: A customized multi-gene panel comprised of Succinate Dehydrogenase Complex Iron Sulfur Subunit B (SDHB), Succinate Dehydrogenase Complex Subunit C (SDHC), Cell Division Cycle 73(CDC73), Calcium Sensing Receptor (CASR), Platelet Derived Growth Factor Receptor Alpha (PDGFRA), Succinate Dehydrogenase Complex Flavoprotein Subunit A (SDHA), Ret Proto-Oncogene (RET), Succinate Dehydrogenase Complex Assembly Factor 2(SDHAF2), Menin 1(MEN1), Succinate Dehydrogenase Complex Subunit D (SDHD), MYC Associated Factor X (MAX) and Protein Kinase CAMP-Dependent Type I Regulatory Subunit Alpha (PRKAR1A) genes was constructed to assess multiple specimen types including: 3 liquid biopsies, 6 FFPE tissues, and 26 peripheral blood samples from 35 unique NET patients. Quality-control and bioinformatics analyses were performed using QCI-Analyze and QClInterpret.

Results: The three liquid biopsies and the 6 FFPE tissue samples were evaluated for somatic mutations; while the 26 peripheral blood samples were analysed using the germ-line pipeline. Five (55.6\%) of the nine patients that were studied for somatic changes carried actionable mutations related to therapy sensitivities. Through the germ-line studies, we observed a $50 \%$ positivity rate for disease predisposition with 16 variants classified according to ACMG (American College of Medical Genetics) Standards and Guidelines.

(Continued on next page)
\end{abstract}

\footnotetext{
* Correspondence: abisgin@yahoo.com

${ }^{+}$Guney Isa Burak and Sonmezler Ozge contributed equally to the article and they both share first authorship of this article.

${ }^{3}$ Cukurova University AGENTEM \& Cukurova University Faculty of Medicine Medical Genetics Department of Balcali Hospital and Clinics, Adana, Turkey Full list of author information is available at the end of the article
}

(c) The Author(s). 2021 Open Access This article is licensed under a Creative Commons Attribution 4.0 International License, which permits use, sharing, adaptation, distribution and reproduction in any medium or format, as long as you give appropriate credit to the original author(s) and the source, provide a link to the Creative Commons licence, and indicate if changes were made. The images or other third party material in this article are included in the article's Creative Commons licence, unless indicated otherwise in a credit line to the material. If material is not included in the article's Creative Commons licence and your intended use is not permitted by statutory regulation or exceeds the permitted use, you will need to obtain permission directly from the copyright holder. To view a copy of this licence, visit http://creativecommons.org/licenses/by/4.0/. The Creative Commons Public Domain Dedication waiver (http://creativecommons.org/publicdomain/zero/1.0/) applies to the data made available in this article, unless otherwise stated in a credit line to the data. 
(Continued from previous page)

Conclusions: Genomic profiling medicine is an emerging area of clinical oncology and has become crucial for disease and patient management by providing a precision approach; this is especially true for rare diseases including rare cancers such as NETs. Notably, this study emphasized the relevance of multiple distinctive biological sample types for use in the genetic testing of cancers to help with the choice of therapy to maximize the likelihood of a positive clinical outcome.

Keywords: Neuroendocrine tumours, Next generation sequencing, Genomic profiling, Somatic mutation, Liquid biopsy, Circulating cell-free DNA

\section{Background}

Neuroendocrine tumours (NETs) are a relatively rare group of cancers which can arise from endocrine and nervous system cells. They can originate from multiple tissues and organs; most frequently from within the lungs, pancreas and intestine. Although the gastroenteropancreatic neuroendocrine tumours group (GEP-NET) is the major class which includes pancreatic neuroendocrine tumours and carcinoids, thymus, parathyroid and lung forms are also take place in rarer cases [1]. The definition and classification of NETs has changed over time due to their complexity [2, 3]. The International Agency for Research on Cancer (IARC) and the World Health Organization (WHO) have published a site-specific criteria and terminology guide to provide uniform classifications This organ-specific approach has aided more accurate nomenclature and grading. Moreover, it has provided us with a better understanding of NETs' organspecific characteristics and prognostic behaviours [4, 5]. Due to the fact that NETs can develop anywhere in the body and that the organ-tissue of origin plays an important role in prognosis and response to treatments, the patients' and the tumour's genetic backgrounds have become more important. The conventional treatment strategies included mainly surgery. However, it is now known that a multidisciplinary approach is necessary for a more personalized and efficient therapy taking into consideration of site of origin, potential of metastasis, grade and the genetic background of the patient. Finally, the heterogeneous origins of NETs lead to a very complicated tumour histology and multiple distinctive aetiologies [6, 7].

Although the vast majority of NETs are sporadic and caused by somatic mutations, germ-line genetic changes also play a critical role in disease inheritance and prognosis $[8,9]$. Therefore, a patient-specific approach is a necessity for disease management [7, 10]. Nextgeneration sequencing (NGS) has become the most powerful methodology for the precise diagnosis, prognosis track and development of therapeutic strategies for many types of cancers. Through the development and testing of customized gene panels for various cancer types, it has been possible to provide precision medicine. In our centre we perform NGS from liquid biopsies one of the most popular topics in oncology - on formalin fixed paraffin embedded (FFPE) tissues, and peripheral blood samples for the accurate genomic profiling of NETs to determine their hereditary predispositions and treatment actionability.

\section{Methods \\ Demographic and clinical information}

Thirty-five distinctive NET patients who were referred to our center for molecular genetic testing were recruited for this study. Patient distribution by their tumor site of origin is as follows; $74.3 \%(n=26)$ foregut (including lung, pancreas, thymus, parathyroid, stomach and medullary thyroid cancer), $5.7 \%(n=2)$ hindgut (rectum cancer), $17.1 \%(n=6)$ other (given in Table 1$)$ and $2.9 \%$ both foregut (pancreas cancer) and hindgut (rectum cancer). All participants signed an informed consent, and were enrolled in accordance with the ethical standards of the institutional ethical committee (Cukurova University Faculty of Medicine Non-Invasive Clinical Research Ethics Commission) and the Helsinki declaration. Starting material types were selected in accordance with the patients' diagnoses, clinical background and familial history. Patients with any inherited syndromes suspected were excluded from the study. Demographic information for the patients is shown in Table 1.

\section{Sampling and DNA isolation}

Liquid biopsy samples were collected via biological sampling tubes special for ccfDNA. Tumor sites were determined to obtain FFPE tissue sections. Somatic DNA from liquid biopsies (circulating cell-free DNA, ccfDNA) and FFPE tissues were isolated by manufacturer's instructions with modifications [QIAamp Circulating Nucleic Acid Kit and DNA FFPE Tissue Kit (Qiagen, Germany)] [11, 12]. Genomic DNA from peripheral blood was isolated via instructions provided by the kit manufacturer [QIAamp DNA Blood Midi Kit (Qiagen, Germany)]. Fluorometric measurements were made using a Qubit 3.0 fluorometer to assess the quality and 
Table 1 Demographic information of the patients, biological sample types, sites of origin and World Health Organization (WHO) classification of NETs

\begin{tabular}{|c|c|c|c|}
\hline Patient & Material & Site of Origin & WHO Classification \\
\hline P1 & Liquid Biopsy & Foregut (Lung) & G1 \\
\hline P2 & Liquid Biopsy & Foregut (Pancreas) & G2 \\
\hline P3 & Liquid Biopsy & Foregut (Pancreas) & G3 \\
\hline P4 & FFPE tissue & Foregut (Thymus) & G1 \\
\hline P5 & FFPE tissue & Other (Surrenal cortical carcinoma, NET) & G3 \\
\hline P6 & FFPE tissue & Hindgut (Rectum) & G2 \\
\hline P7 & FFPE tissue & Foregut (Thyroid) & G1 \\
\hline P8 & FFPE tissue & Foregut (Pancreas) & G3 \\
\hline P9 & FFPE tissue & Other (Urinary system, NEC) & G3 \\
\hline P10 & Peripheral Blood & Foregut (Thyroid) & G1 \\
\hline P11 & Peripheral Blood & Foregut (Parathyroid) & G1 \\
\hline P12 & Peripheral Blood & Foregut (Thyroid) & G1 \\
\hline P13 & Peripheral Blood & Foregut (Pancreas) & G3 \\
\hline P14 & Peripheral Blood & Foregut (Stomach) & G2 \\
\hline P15 & Peripheral Blood & Other (Primary location unknown) & G3 \\
\hline P16 & Peripheral Blood & Foregut (Thyroid) & G1 \\
\hline P17 & Peripheral Blood & Foregut (Thyroid) & G1 \\
\hline P18 & Peripheral Blood & Foregut (Thyroid) & G2 \\
\hline P19 & Peripheral Blood & Foregut (Thyroid) & G1 \\
\hline P20 & Peripheral Blood & Hindgut (Rectum) & G3 \\
\hline P21 & Peripheral Blood & Foregut (Thyroid) & G1 \\
\hline P22 & Peripheral Blood & Foregut (Thyroid) & G2 \\
\hline P23 & Peripheral Blood & Foregut (Pancreas) & G3 \\
\hline P24 & Peripheral Blood & Foregut (Pancreas) & G2 \\
\hline P25 & Peripheral Blood & Foregut (Pancreas) & G1 \\
\hline P26 & Peripheral Blood & Foregut (Pancreas) & G1 \\
\hline P27 & Peripheral Blood & Foregut (Pancreas) + Hindgut (Rectum) & G3 \\
\hline P28 & Peripheral Blood & Foregut (Pancreas) & G3 \\
\hline P29 & Peripheral Blood & Foregut (Pancreas) & G1 \\
\hline P30 & Peripheral Blood & Other (Primary location unknown, Hepatic and Pancreas NET) & G3 \\
\hline P31 & Peripheral Blood & Foregut (Pancreas) & G1 \\
\hline P32 & Peripheral Blood & Foregut (Pancreas) & G3 \\
\hline P33 & Peripheral Blood & Foregut (Pancreas) & G1 \\
\hline P34 & Peripheral Blood & Other (Head and Neck NET) & G3 \\
\hline P35 & Peripheral Blood & Other (Paraganglioma, NET) & G2 \\
\hline
\end{tabular}

G Grade, NET Neuroendocrine Tumour, NEC Neuroendocrine Carcinoma. Age range in males: 17-76. Age range in females: 15-77

quantity of the isolated DNAs. The purified DNAs were then subjected to next-generation sequencing.

\section{Next-generation sequencing and QC}

Target enrichment was performed starting with $40 \mathrm{ng}$ of input gDNA from PBMCs, 100 ng of DNA from peripheral blood ccfDNA from liquid biopsies; and $250 \mathrm{ng}$ of DNA from FFPE samples using a customized-designed multi-gene capture panel (Qiagen, Hilden, Germany) that consists of 12 NET-related genes. All exons and exon-intron junctions of Succinate Dehydrogenase Complex Iron Sulfur Subunit B (SDHB), Succinate Dehydrogenase Complex Subunit C (SDHC), Cell Division Cycle 73 (CDC73), Calcium Sensing Receptor (CASR), Platelet Derived Growth Factor Receptor Alpha (PDGFRA), Succinate Dehydrogenase Complex Flavoprotein Subunit A 
Table 2 Distribution of the actionable variants which all are in confidentiality rates according to the literature and our previous clinical validation studies [11-13]

\begin{tabular}{|c|c|c|c|c|c|}
\hline & Patient & Anatomic location & Gene & Variant (amino acid change) & Allel frequency (\%) \\
\hline \multirow[t]{4}{*}{ Liquid Biopsy } & P2 & Pancreas & RET & D698fs*71 & 0.65 \\
\hline & & & & N950fs*9 & 0.54 \\
\hline & P3 & Pancreas & RET & N950fs*9 & 1.63 \\
\hline & & & & L19del & 1.48 \\
\hline \multirow[t]{6}{*}{ FFPE tissue } & P4 & Other & PDGFRA & T463S & 49 \\
\hline & P5 & Other & RET & L390G & 0.94 \\
\hline & & & SDHA & $\mathrm{G} 112 \mathrm{fs}^{*} 49$ & 0.72 \\
\hline & P9 & Other & SDHA & $\mathrm{K} 541^{*}$ & 5.8 \\
\hline & & & & R465Q & 14 \\
\hline & & & & S456L & 28 \\
\hline
\end{tabular}

$F$ Female, $M$ Male

* symbol were used to indicate stop codon formation as recommended in HUGO Gene Nomenclature Committee standards

(SDHA), Ret Proto-Oncogene (RET), Succinate Dehydrogenase Complex Assembly Factor 2 (SDHAF2), Menin 1 (MEN1), Succinate Dehydrogenase Complex Subunit D $(S D H D)$, MYC Associated Factor X $(M A X)$ and Protein Kinase CAMP-Dependent Type I Regulatory Subunit Alpha (PRKAR1A) genes were amplified and sequenced with a minimum of 300x coverage for germ-line studies and 1500x coverage for somatic studies.

Sequencing quality control assessments were carried out using the QCI-Analyze tool, and the QCI-Interpret interface was used for the bioinformatics analyses. Multiple quality control parameters including total yield, variant frequency, forward/reverse ratio, depth of coverage and quality score were assessed for each sample type. All of the detected genetic changes were evaluated independently of the patients' diagnoses. Next generation sequencing steps and quality control assessments were performed with optimization based on previous studies [11, 12].

\section{Data interpretation and bioinformatics analysis}

Bioinformatics analyses were made for each variant detected using multiple databases including: HGMD ( $\mathrm{Hu}-$ man Gene Mutation Database), ClinVar, NCBI (National Center for Biotechnology Information), VarSome (The

Table 3 Implemented variant classification criteria of detected germ-line mutations

\begin{tabular}{llll}
\hline Patient & Gene & Variant (amino acid change) & $\begin{array}{c}\text { ACMG criteria } \\
\text { (doi: 10.1038/gim.2015.30) }\end{array}$ \\
\hline P11 & KIT & M541L (Heterozygote) & PM2, BP4, BP6 \\
& SDHD & G12S (Heterozygote) & PP2, BS1, BS3, BP4, BP6 \\
P14 & SDHB & G19FS 57 (Heterozygote) & PVS1, PM1, PM2 \\
P15 & PDGFRA & S66R (Heterozygote) & PM2, BP1, BP4 \\
P17 & RET & G691S (Heterozygote) & PP2, BA1, BS3, BP4 \\
P18 & RET & G691S (Heterozygote) & PP2, BA1, BS3, BP4 \\
P19 & RET & G691S (Heterozygote) & PP2, BA1, BS3, BP4 \\
P22 & RET & G691S (Heterozygote) & PP2, BA1, BS3, BP4 \\
& KIT & A431E (Heterozygote) & PM1, PM2, BP4 \\
P24 & RET & G691S (Homozygote) & PP2, BA1, BS3, BP4 \\
& RET & A1051V (Heterozygote) & PM1, PP2, PP3 \\
P25 & RET & G691S (Heterozygote) & PP2, BA1, BS3, BP4 \\
P29 & RIT & R946* (Heterozygote) & PVS1, PP3, BS2 \\
P30 & RET & A96V (Heterozygote) & PM2, PP2, BP4 \\
P34 & KIT & M541L (Heterozygote) & PM2, BP4, BP6 \\
P35 & SDHC & R72H (Heterozygote) & PM1, PM2, PP3 \\
\hline
\end{tabular}

PVS1 Pathogenic Very Strong, PP2 and PP3 Pathogenic Supporting, PM1 and PM2 Pathogenic Moderate, BP1, BP4 and BP6 Benign Supporting, BA1and BS3 Benign Strong

* symbol were used to indicate stop codon formation as recommended in HUGO Gene Nomenclature Committee standards 
Human Genomic Variant Search Engine), ExAC (The Exome Aggregation Consortium), 1000 Genome Frequency, ESP (Exome Sequencing Project), Ancestry, Ingenuity Knowledge Base, COSMIC, GnomAD, OMIM (Online Mendelian Inheritance in Man) and Franklin to provide as much information as possible for making each clinical interpretation. All of the in-silico predictions were performed using a minimum of 10 different analysis tools including MutationTaster, SIFT and PolyPhen 2. The pathogenicity classification of each of the detected variants was determined in compliance with ACMG guidelines and standards.

In the secondary analysis, all of the variants which met the quality control criteria were investigated in accordance with the patients' diagnoses and clinical findings. Hereditary predispositions were determined for germ-line studies, and actionability was assessed for the detected somatic variants.

\section{Results}

Total of thirty-five patients were recruited to the study which $25.7 \%(n=9)$ of them are for somatic and $74.3 \%$ $(n=26)$ of them are for germline molecular testing. Six patients of 9 somatic studies were analysed for somatic variants in FFPE samples with their NET diagnoses. Actionable mutations with regard to therapy were detected in $3(50 \%)$ of them. Patients had six actionable mutations in PDGFRA, RET and SDHA genes. Among the liquid biopsy studies, we detected four actionable mutations within the $R E T$ oncogene in two $(75 \%)$ of the three liquid biopsy specimens. Mutations were observed in the same clone for one of these patients while the other patient had the mutation in different clones. The distribution of the detected somatic alterations is given in Table 2 . The overall positivity rate was $55.6 \%$ among the somatic studies. Additionally, somatic status of PDGFR variant with $49 \%$ allel frequency was confirmed by the re-evaluation with germ-line study.

Through the germ-line studies, we also observed a $50 \%$ positivity rate for disease predisposition with 16 variants identified among 13 patients. Germ-line mutations were seen in the RET, KIT, SDHB, SDHC, SDHD and PDGFRA genes. The variant distributions and their

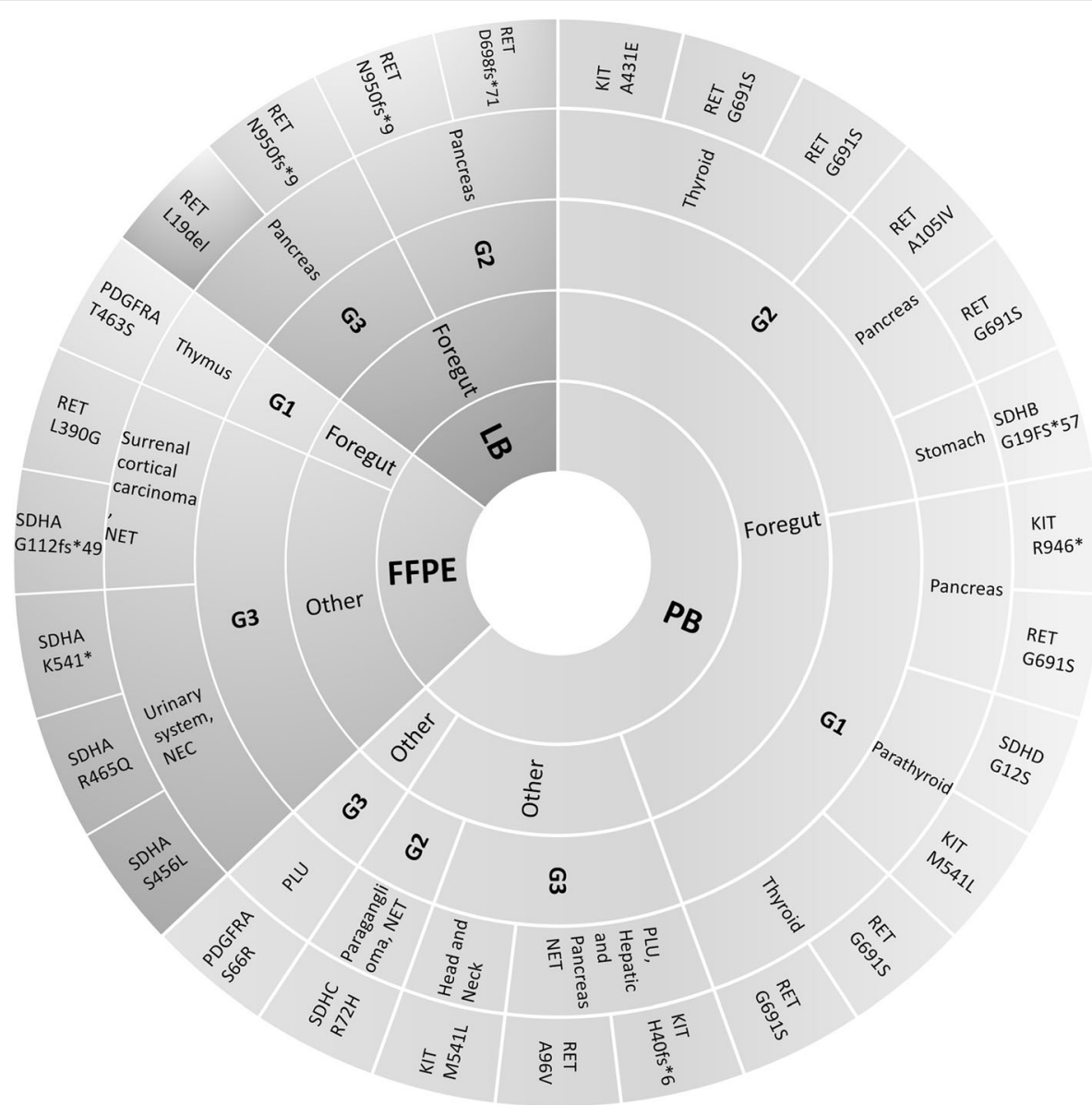

Fig. 1 Variant distribution versus clinical characteristics. PB: Peripheral Blood, LB: Liquid Biopsy, FFPE: Formalin Fixed Paraffin Embedded Tissue, G: Grade, PLU: Primary Location Unknown, NET: Neuroendocrine Tumour, NEC: Neuroendocrine Carcinoma 
classification criteria are listed in Table 3 according to the ACMG Standards and Guidelines.

Additionally, variation quantiles according to grade and site of origin were given in Fig. 1. The overall mutation distribution was not distinctive between neither the different material types nor the severity of diseases.

This study evaluated both the risk factors and proposed potential risk factors together with germline and somatic genomic profiling. A multi-gene panel was used to investigate the genetics underlying both germline and somatic NETs and was demonstrated to provide actionable information in greater than $50 \%$ of the cases including those were evaluated using liquid biopsy specimens.

\section{Discussion}

Comprehensive molecular genetic profiling is a must for heterogeneous group of cancers such as NETs. As a result, we detected somatic variations in RET, PDGFRA and SDHA genes; while RET, KIT, SDHB, SDHC, SDHD and PDGFRA genes were mutated in germ-line studies. The distribution of the detected variants and genes are deviational because of the complex tissue origins of NETs and the individual differences. When the genetic changes classified by the ACMG criteria and assessed together with the both biological sample type and pathological findings, the frequency becomes similar between the groups. A comprehensive grade specific study with a broaden cohort is needed due to more precise variant interpretation and molecular grade classification of NETs.

We also emphasized that not only the somatic changes play a role in cancer progression and therapeutic approach, but a complement study of germ-line genetic profiling is beneficial for the patient. Cote et al. has shown that the liquid biopsy plays a prognostic role on medullary thyroid carcinoma due to a single-variant study of $R E T$ oncogene, and can be efficiently used in monitoring the disease [14]. Cavalcanti et al. recently reported that tracking PDGFRA expressions provide as a promising anti-angiogenic target in welldifferentiated NETs [15]. Knösel et al. also showed that the over-expression of KIT and PDGFRA genes are related to short survival and a negative prognostic factor in advanced pancreatic neuroendocrine tumours [16]. Niemeijer et al. reported that SDH mutations also cause other types of cancers which have neuroendocrine origins other than paraganglionic tumours. Literature of NETs points that a broaden genetic profiling of both somatic and germline mutations should be the next step to improve NET diagnostics and therapeutic approaches. Emerging the accurate material type with the proper multi-gene panels that next generation sequencing technologies offer is the key.

\section{Conclusions}

Molecular genetic testing strategies can be tailored to fit an individual patient's specific needs. The most efficient approach including the best specimen type and target gene panel will differ for each patient group, particularly those that are rare and/or heterogeneous cancers such as NETs. Genomic profiling medicine can act as a bridge between clinicians and patients for to provide precision for the development of therapeutic algorithms. Additionally, it is also crucial to practice effective patient follow-up to ensure proper patient management for both the patients and their families.

In summation, in this study we show the significance of selecting determinative biological samples in the decision making process to provide the best possible health care service.

\section{Abbreviations}

NET: Neuroendocrine Tumours; NGS: Next Generation Sequencing; GEPNET: Gastroenteropancreatic Neuroendocrine Tumours;

DNA: Deoxyribonucleic Acid; ccfDNA: Circulating cell-free DNA; gDNA: Genomic DNA; FFPE: Formalin Fixed Paraffin Embedded; ACMG: American College of Medical Genetics and Genomics;

SDHB: Succinate Dehydrogenase Complex Iron Sulfur Subunit B;

SDHC: Succinate Dehydrogenase Complex Subunit C; CDC73: Cell Division

Cycle 73; CASR: Calcium Sensing Receptor; PDGFRA: Platelet Derived Growth Factor Receptor Alpha; SDHA: Succinate Dehydrogenase Complex

Flavoprotein Subunit A; RET: Ret Proto-Oncogene; SDHAF2: Succinate Dehydrogenase Complex Assembly Factor 2; MEN1: Menin 1;

SDHD: Succinate Dehydrogenase Complex Subunit D; MAX: MYC Associated Factor X; PRKAR1A: Protein Kinase CAMP-Dependent Type I Regulatory Subunit Alpha; HGMD: Human Gene Mutation Database; NCBI: National Center for Biotechnology Information); ExAC: The Exome Aggregation Consortium; ESP: Exome Sequencing Project; COSMIC: Catalogue of Somatic Mutations; OMIM: Online Mendelian Inheritance in Man; ACMG: American College of Medical Genetics; IARC: International Agency for Research on Cancer; WHO: World Health Organization

\section{Acknowledgements}

We would like to thank the editorial board of "BMC Cancer" for their review. We also thank to enrolled patients and their families for participation.

\section{Authors' contributions}

All authors have read and approved the manuscript. Author contributions were as follows; conceptualization, investigation and writing-original draft by IBG; investigation, methodology, validation, visualization and writing-original draft by OS; investigation, methodology, visualization and data curation by $\mathrm{CM}$; patient recruitment and clinical interpretation by GB and ZYD;

conceptualization, project administration, supervision and writing - review \& editing by $A B$.

\section{Funding}

This study was partially supported for the establishment of next-generationsequencing custom panel designs and pipelines for the liquid biopsy samples by grants from Cukurova University Scientific Research Projects (Project No: TAY-2017-8767). The funding source had no role in publication.

\section{Availability of data and materials}

The datasets used and/or analysed during the current study are available from the corresponding author upon reasonable request.

\section{Declarations}

Ethics approval and consent to participate

All participants were informed, and signed consent/permissions for this research in accordance with Helsinki declaration. The parental consent form was obtained from the children included in this study. Ethics approval was received from Cukurova University Ethical Committee. 


\section{Consent for publication}

Not applicable.

\section{Competing interests}

The authors declare that they have no competing interests.

\section{Author details}

'Cukurova University Faculty of Medicine Nuclear Medicine Department of Balcali Hospital and Clinics, Adana, Turkey. ${ }^{2}$ Cukurova University AGENTEM (Adana Genetic Diseases Diagnosis and Treatment Center), Adana, Turkey. ${ }^{3}$ Cukurova University AGENTEM \& Cukurova University Faculty of Medicine Medical Genetics Department of Balcali Hospital and Clinics, Adana, Turkey.

Received: 4 June 2020 Accepted: 24 February 2021

Published online: 06 March 2021

\section{References}

1. Oronsky B, Ma PC, Morgensztern D, Carter CA. Nothing But NET: a review of neuroendocrine tumors and carcinomas. Neoplasia. 2017;19(12):991-1002.

2. Shah MH, Goldner WS, Halfdanarson TR, Bergsland E, Berlin JD, Halperin D, et al. NCCN guidelines insights: neuroendocrine and adrenal tumors, version 2.2018. J Natl Compr Cancer Netw. 2018;16(6):693-702.

3. Basu B, Sirohi B, Corrie P. Systemic therapy for neuroendocrine tumours of gastroenteropancreatic origin. Endocr Relat Cancer. 2010;17(1):R75-90.

4. Rindi G, Klimstra DS, Abedi-Ardekani B, Asa SL, Bosman FT, Brambilla E, et al. A common classification framework for neuroendocrine neoplasms: an International Agency for Research on Cancer (IARC) and World Health Organization (WHO) expert consensus proposal. Mod Pathol. 2018;31(12): 1770-86.

5. Mafficini A, Scarpa A. Genomic landscape of pancreatic neuroendocrine tumours: the international Cancer genome consortium. J Endocrinol. 2018; 236(3):R161-r7.

6. Tsoli M, Chatzellis E, Koumarianou A, Kolomodi D, Kaltsas G. Current best practice in the management of neuroendocrine tumors. Ther Adv Endocrinol Metab. 2019;10:2042018818804698.

7. Vijayvergia N, Boland PM, Handorf E, Gustafson KS, Gong Y, Cooper HS, et al. Molecular profiling of neuroendocrine malignancies to identify prognostic and therapeutic markers: a fox Chase Cancer Center pilot study. Br I Cancer. 2016;115(5):564-70.

8. Mafficini A, Scarpa A. Genetics and epigenetics of Gastroenteropancreatic neuroendocrine neoplasms. Endocr Rev. 2019;40(2):506-36.

9. Banck MS, Kanwar R, Kulkarni AA, Boora GK, Metge F, Kipp BR, et al. The genomic landscape of small intestine neuroendocrine tumors. J Clin Invest. 2013;123(6):2502-8.

10. Astsaturov IA, Cohen SJ, Engstrom PF, Millis SZ. Profiling of 1,350 neuroendocrine tumors for identification of multiple potential drug targets. J Clin Oncol. 2014;32(15_suppl):4113.

11. Boga I, Sonmezler O, Bisgin A. Clinical Validation of a Novel GeneReader Next Generation Sequencing System for Tumor Specific Mutations and Bioinformatics Variant Analysis. Clin Lab. 2020;66(11):2213-8.

12. Sonmezler O, Boga I, Bisgin A. Integration of Liquid Biopsies into Clinical Laboratory Applications via NGS in Cancer Diagnostics. Clin Lab. 2020;66(5): 763-9.

13. Tang $Y$, Liu $X, O u$ ZA, He Z, Zhu Q, Wang $Y$, et al. Maximum allele frequency observed in plasma: a potential indicator of liquid biopsy sensitivity. Oncol Lett. 2019;18(2):2118-24.

14. Cote GJ, Evers C, Hu Ml, Grubbs EG, Williams MD, Hai T, et al. Prognostic significance of circulating RET M918T mutated tumor DNA in patients with advanced medullary thyroid carcinoma. J Clin Endocrinol Metab. 2017; 102(9):3591-9.

15. Cavalcanti E, Ignazzi A, De Michele F, Caruso ML. PDGFRa expression as a novel therapeutic marker in well-differentiated neuroendocrine tumors. Cancer Biol Ther. 2019;20(4):423-30.

16. Knösel T, Chen Y, Altendorf-Hofmann A, Danielczok C, Freesmeyer M, Settmacher U, et al. High KIT and PDGFRA are associated with shorter patients survival in gastroenteropancreatic neuroendocrine tumors, but mutations are a rare event. J Cancer Res Clin Oncol. 2012;138(3):397-403.

\section{Publisher's Note}

Springer Nature remains neutral with regard to jurisdictional claims in published maps and institutional affiliations.

\section{Ready to submit your research? Choose BMC and benefit from:}

- fast, convenient online submission

- thorough peer review by experienced researchers in your field

- rapid publication on acceptance

- support for research data, including large and complex data types

- gold Open Access which fosters wider collaboration and increased citations

- maximum visibility for your research: over $100 \mathrm{M}$ website views per year

At BMC, research is always in progress.

Learn more biomedcentral.com/submissions 